\title{
CHARACTERIZATIONS OF STRICTLY MONOTONE SETS
}

\author{
JEAN CHAN STANEK
}

\begin{abstract}
One natural generalization of convex sets is the concept of strictly monotone sets. In this paper, Tietze's characterization theorem on convex sets is generalized to strictly monotone sets. The main result is that a closed connected set in the Euclidean plane $E_{2}$ is strictly monotone if and only if it is locally strictly monotone. This theorem is shown to hold in $E_{n}$ with additional hypotheses.
\end{abstract}

1. Introduction. Tietze [4] proved that a closed connected locally convex set in $E_{n}$ is convex. This theorem was extended by Klee [1] to a topological linear space. In this paper, we investigate strictly monotone sets which include convex sets as special cases. Applying a result on stripwise subconvex sets [2], we prove that a closed connected set in $E_{2}$ is strictly monotone if and only if it is locally strictly monotone. The concept of strictly monotone sets can be extended to $E_{n}$ and a characterization theorem on strictly monotone sets in $E_{n}$ is established with additional hypotheses. For simplicity, we limit our discussion to $E_{n}$, but all the concepts and theorems in the following are valid in arbitrary topological linear spaces over the real field.

2. Preliminaries. We follow the notations in Valentine [5]. The closure, interior, boundary, and convex hull of a set $S \subset E_{n}$ are denoted by cl $S$, int $S$, bd $S$, and conv $S$, respectively. If $x$ and $y$ are distinct points in $E_{n}$, then $x y$ denotes the closed line segment joining $x$ and $y$ and intv $x y$ denotes the relative interior of $x y$.

Represent $E_{2}$ as a Euclidean product space $\left(E_{1}, R\right)$ where $E_{1}$ and $R$ are one-dimensional subspaces of $E_{2}$, and assign positive and negative directions to $R$. A line parallel to $R$ is called a vertical line and a line parallel to $E_{1}$ is called a horizontal line. If $x$ and $y$ are points in $\left(E_{1}, R\right)$ not lying on a vertical or horizontal line, the line segment $x y$ determines two closed convex triangles having $x y$ as one side and each of the remaining sides parallel to $E_{1}$ and $R$, respectively. The triangle below $x y$, denoted by $T(x, y)$, is called the lower triangle determined by $x$ and $y$. If $x$ and $y$ are on a vertical or horizontal line, $T(x, y)$ is taken to be $x y$. A convex arc $C(x, y)$ joining $x$ and $y$ is called a monotone arc if $C(x, y) \subset T(x, y)$. We now describe monotone sets and strictly monotone sets as follows.

Received by the editors August 11, 1976.

AMS (MOS) subject classifications (1970). Primary 52-00, 52A10, 52A20. sets.

Key words and phrases. Convex sets, Tietze's theorem, strictly monotone sets, stripwise convex 
Definition 1. A set $S \subset\left(E_{1}, R\right)$ is monotone if for each pair of distinct points $x \in S$ and $y \in S$ it is true that there exists a monotone arc $C(x, y)$ joining $x$ and $y$ in $S$. The set $S$ is strictly monotone if it is monotone and if the convex arc $C(x, y)$ does not contain a horizontal line segment when $x$ and $y$ are not on a horizontal line.

Note that when $x$ and $y$ are on a vertical or horizontal line, the monotone arc $C(x, y)$ reduces to $x y$. Thus a monotone or strictly monotone set is convex along vertical and horizontal directions.

Definition 2. A set $S \subset\left(E_{1}, R\right)$ is locally (strictly) monotone if for each $x \in S$ there exists a neighborhood $N$ of $x$ such that $N \cap S$ is (strictly) monotone.

It is easy to see that the closure of a monotone set is monotone and that an open monotone set is strictly monotone. Note that it is not true in general that a locally monotone set is monotone. For example, consider the set $S=\{a b \cup b c \cup c d\}$, as shown in Figure 1. Clearly $S$ is locally monotone but it is not monotone since there exists no monotone arc in $S$ to join the points $a$ and $d$. It is, however, possible to establish characterizations on strictly monotone sets. In order to prove our results, we apply a theorem on stripwise subconvex sets [2]. The relevant definitions and theorem are stated below.

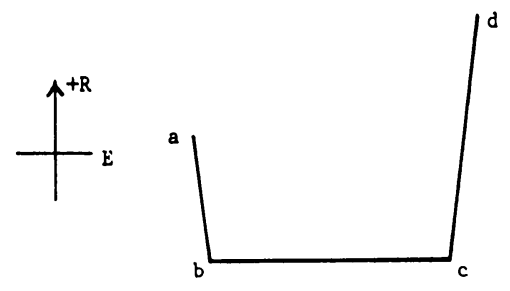

FIGURE 1

Definition 3. A set $S \subset\left(E_{1}, R\right)$ is stripwise subconvex if for each pair of distinct points $x$ and $y$ in $S$ there exists a convex arc $A(x, y)$ in $S$ joining $x$ and $y$ such that:

(1) if $x$ and $y$ are on a vertical line, then $A(x, y)=x y$; and

(2) if $x$ and $y$ are not on a vertical line, then $A(x, y)$ lies in the closed lower half-plane bounded by the line through $x$ and $y$ and $A(x, y)$ lies in the convex hull of the vertical lines through $x$ and $y$, respectively. Such an $\operatorname{arc} A(x, y)$ is called a stripwise subconvex arc.

The set $S$ is locally stripwise subconvex if for each $x \in S$ there exists a neighborhood $N$ of $x$ such that $N \cap S$ is stripwise subconvex.

THEOREM 1. A closed connected set in $\left(E_{1}, R\right)$ is stripwise subconvex if and only if it is locally stripwise subconvex [2, Theorem 4.2, p. 93].

A strictly monotone set is clearly a stripwise subconvex set but the converse is not true in general. 


\section{The results.}

TheOREM 2. Let $S \subset\left(E_{1}, R\right)$ be a closed connected set. The set $S$ is strictly monotone if and only if it is locally strictly monotone.

Proof. The necessity is trivial. To prove the sufficiency, let $x$ and $y$ be arbitrary distinct points of $S$. Since $S$ is locally strictly monotone, it is locally stripwise subconvex. By Theorem 1 , the set $S$ is stripwise subconvex. Let $A(x, y)$ be a stripwise subconvex arc joining $x$ and $y$ in $S$. Since $S$ is closed, it follows that we may take the stripwise subconvex $\operatorname{arc} A(x, y)$ to be minimal in the sense that the convex hull of any other stripwise subconvex arc joining $x$ and $y$ in $S$ contains the set conv $A(x, y)$. We will show that $A(x, y)$ is either $x y$ or a monotone arc not containing a horizontal line segment. Consider the following cases:

(1) The points $x$ and $y$ are not on a vertical or horizontal line. We begin by showing $A(x, y) \subset T(x, y)$. Suppose $A(x, y) \not \subset T(x, y)$ and assume $y$ lies in the upper half-plane bounded by the line through $x$ and parallel to $E_{1}$. Let $L$ be a horizontal line of support of conv $A(x, y)$. First suppose that $L \cap$ conv $A(x, y)=\{z\}$ where $z$ is an exposed point of conv $A(x, y)$. Since $S$ is locally strictly monotone at $z$, there exists a neighborhood $N$ of $z$ such that $N \cap S$ is strictly monotone. We may choose $N$ to be convex with $x \notin N$ and $y \notin N$. Using arguments similar to those of part (a) in the proof of Theorem 2 in [3], we can find points $r$ and $s$ in $N \cap A(x, y) \subset N \cap S$ such that the line segment $r s$ is nondegenerate and such that $r$ and $s$ lie on a line parallel to $L$. It follows from Definition 1 that $r s \subset N \cap S$. Let

$$
C(x, y)=A(x, r) \cup r s \cup A(s, y) \subset S
$$

where $A(x, r)$ and $A(s, y)$ are the subarcs of $A(x, y)$ joining the indicated pairs of points. Clearly $C(x, y)$ is a stripwise subconvex arc and conv $A(x, y)$ $\ell$ conv $C(x, y)$. The existence of $C(x, y)$ contradicts the minimality of $A(x, y)$. Hence $A(x, y) \subset T(x, y)$.

Next we consider the case where $L \cap A(x, y)$ is a nondegenerate line

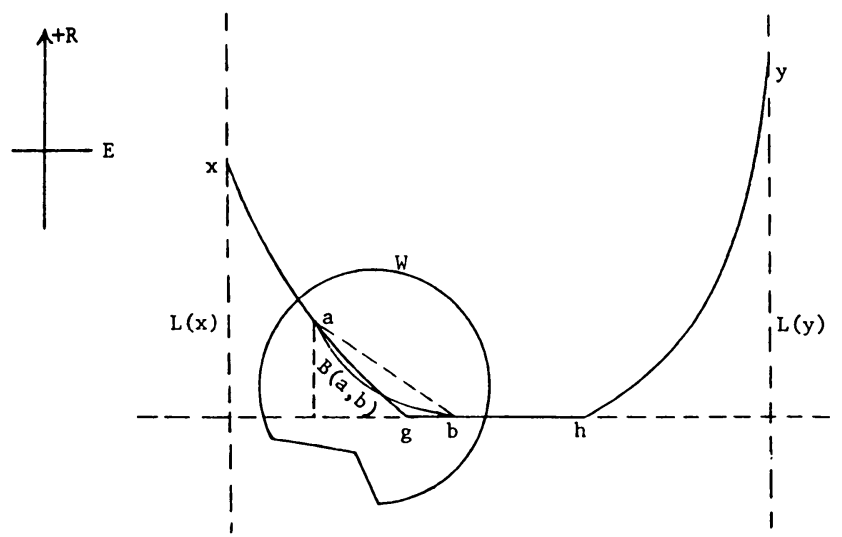

FIGURE 2 
segment $g h$. Since $A(x, y) \not T(x, y)$, we have $x \notin g h$ and $y \notin g h$. For definiteness, we assume that $g \in A(x, h)$ where $A(x, h)$ is the subarc of $A(x, y)$ joining $x$ and $h$. See Figure 2 .

Since $S$ is locally strictly monotone, there exists a neighborhood $W$ of $g$ such that $W \cap S$ is strictly monotone. We may choose $W$ with $x \notin W$ and $h \notin W$. Choose a point $a \in A(x, g) \cap W$ with $a \neq g$, where $A(x, g)$ is the subarc of $A(x, y)$ joining $x$ and $g$. Choose a point $b \in($ intv $g h) \cap W$. Since $a$ and $b$ are in $W \cap S$ not lying on a horizontal line, there exists a monotone $\operatorname{arc} B(a, b)$ with no horizontal line segment joining $a$ and $b$ in $W \cap S$. Let

$$
B_{0}(a, b)=\operatorname{bd}\{\operatorname{conv} B(a, b) \cap \operatorname{conv} A(x, y)\}-\operatorname{intv} a b .
$$

It is clear that $B_{0}(a, b) \subset S$. Let

$$
A_{0}(x, y)=A(x, a) \cup B_{0}(a, b) \cup A(b, y),
$$

where $A(x, a)$ and $A(b, y)$ are the subarcs of $A(x, y)$ joining $x$ to $a$ and $b$ to $y$, respectively. The compact set bounded by $x y \cup A_{0}(x, y)$ has a nonempty interior, and clearly it has a line of support through each point of $x y \cup$ $A_{0}(x, y)$. It follows from a characterization theorem on closed convex sets [5, Theorem 4.1, p. 47] that $A_{0}(x, y)$ is a convex arc. Hence $A_{0}(x, y)$ is a stripwise subconvex arc joining $x$ and $y$ in $S$. Note that $g \notin A_{0}(x, y)$, since $B(a, b)$ does not contain a horizontal line segment. Thus conv $A(x, y) \not$ conv $A_{0}(x, y)$, contradicting the minimality of $A(x, y)$. Therefore $A(x, y) \subset$ $T(x, y)$.

It remains to show that $A(x, y)$ does not contain a nondegenerate horizontal line segment. If $A(x, y)$ has a maximal horizontal line segment $x d, x \neq d$, then there exists a neighborhood $M$ of $d$ such that $M \cap S$ is strictly monotone with $x \notin M$ and $y \notin M$. Choose a point $k \in($ intv $x d) \cap M$ and a point $c \in A(d, y) \cap M$ with $c \neq d$, where $A(d, y)$ is the subarc of $A(x, y)$ joining $d$ and $y$. See Figure 3. Since $k$ and $c$ are in $M \cap S$, not lying on a horizontal line, there exists a monotone $\operatorname{arc} B_{2}(k, c)$ with no horizontal line segment joining $k$ and $c$ in $S$. Let

$$
B_{1}(k, c)=\text { bd }\left\{\operatorname{conv} B_{2}(k, c) \cap \operatorname{conv} A(k, c)\right\}-\operatorname{intv} k c,
$$

where $A(k, c)$ is the subarc of $A(x, y)$ joining $k$ and $c$. Clearly $B_{1}(k, c) \subset S$. Let

$$
A_{1}(x, y)=x k \cup B_{1}(k, c) \cup A(c, y),
$$

where $A(c, y)$ is the subarc of $A(x, y)$ joining $c$ and $y$. The $\operatorname{arc} A_{1}(x, y)$ is convex and it is a subset of $S \cap T(x, y)$. Thus $A_{1}(x, y)$ is a monotone arc joining $x$ and $y$ in $S$. Note that $d \notin A_{1}(x, y)$ since $d \notin B_{2}(k, c)$. Thus conv $A(x, y) \not \subset$ conv $A_{1}(x, y)$, contradicting the minimality of $A(x, y)$. Hence $A(x, y)$ does not contain a nondegenerate horizontal line segment. Thus $A(x, y)$ is the required monotone arc joining $x$ and $y$ in $S$ not containing a horizontal line segment. 

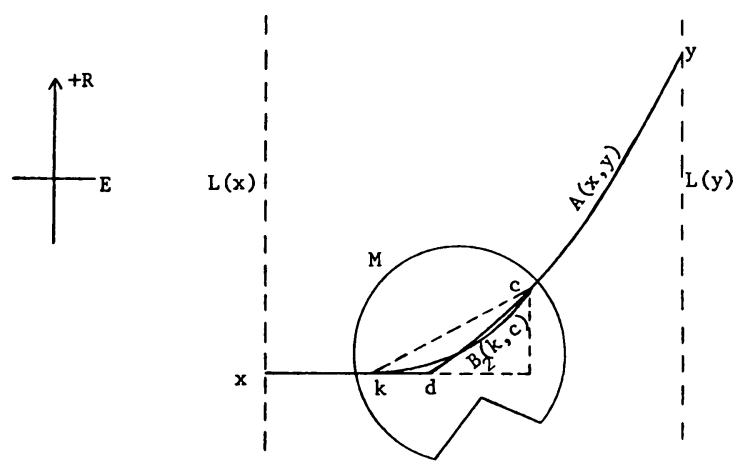

FIGURE 3

(2) The points $x$ and $y$ lie on a vertical line. Then $A(x, y)=x y \subset S$.

(3) The points $x$ and $y$ lie on a horizontal line. Let $L$ be a horizontal line of support to conv $A(x, y)$. If $A(x, y) \neq x y$, we derive a contradiction to the minimality of $A(x, y)$ as in (1). Hence $A(x, y)=x y$ must hold. This completes the proof of Theorem 2.

The concept of strictly monotone sets can be extended to higher dimensional Euclidean spaces. Represent $E_{n}$ as a Euclidean product space $\left(E_{n-1}, R\right)$ where $E_{n-1}$ is an $(n-1)$-dimensional subspace of $E_{n}$ and $R$ is a one-dimensional subspace of $E_{n}$ with positive and negative directions assigned. If $F$ is a two-dimensional flat of $\left(E_{n-1}, R\right)$ parallel to $R, F$ is represented as a translate of a subspace $(E, R)$, and $E$ a one-dimensional subspace of $E_{n-1}$. The concepts of lower triangles, vertical lines, horizontal lines and monotone arcs are valid in $F$.

Definition 4. A set $S \subset\left(E_{n-1}, R\right)$ is strictly monotone if for each pair of distinct points $x \in S$ and $y \in S$ there exists a two-dimensional flat $F$ of $\left(E_{n-1}, R\right)$ parallel to $R$ containing $x$ and $y$ such that:

(1) there exists a monotone arc $C(x, y)$ joining $x$ and $y$ in $F \cap S$ where $C(x, y)$ does not contain a horizontal line segment if $x$ and $y$ are not on a horizontal line in $F$; and

(2) $x y \subset F \cap S$ if $x$ and $y$ are on a horizontal line in $F$.

The set $S$ is locally strictly monotone if for each $x \in S$ there exists a neighborhood $N$ such that $N \cap S$ is strictly monotone.

A generalization of Theorem 2 can be obtained in $\left(E_{n-1}, R\right)$, using the concept of stripwise subconvexity in $\left(E_{n-1}, R\right)$ and Theorem 3 [2, Theorem 4.3 , p. 98 ].

Definition 5. A set $S \subset\left(E_{n-1}, R\right)$ is stripwise subconvex if for each pair of distinct points $x \in S$ and $y \in S$ there exists a two-dimensional flat $F$ of $\left(E_{n-1}, R\right)$ parallel to $R$ and a stripwise subconvex arc $C(x, y)$ joining $x$ and $y$ such that $C(x, y) \subset F \cap S$. Local stripwise subconvexity is defined accordingly.

TheOREM 3. Let $S \subset\left(E_{n-1}, R\right)$ be a closed connected set which is bounded below and assume that every line parallel to $R$ intersects $S$ in a connected set. 
Then the set $S$ is stripwise subconvex if and only if it is locally stripwise subconvex.

By an application of Theorem 3 and the arguments given in the proof of Theorem 2, we derive the following result.

THEOREM 4. Let $S \subset\left(E_{n-1}, R\right)$ be a closed connected set which is bounded below and assume that every line parallel to $R$ intersects $S$ in a connected set. Then the set $S$ is strictly monotone if and only if it is locally strictly monotone.

Proof. The necessity is obvious. To prove the sufficiency, let $x$ and $y$ be arbitrary distinct points of $S$. Since $S$ is locally strictly monotone, it is locally stripwise subconvex; and therefore it is stripwise subconvex by Theorem 3 . Let $F$ be a two-dimensional flat of $\left(E_{n-1}, R\right)$ parallel to $R$ containing $x$ and $y$, and let $C(x, y)$ be a stripwise subconvex arc joining $x$ and $y$ in $F \cap S$. The existence of $C(x, y)$ in $F \cap S$ implies that $x$ and $y$ lie in the same component $D$ of $F \cap S$. The component $D$ is closed since $F \cap S$ is closed. As in the proof of Theorem 2, we take $C(x, y)$ to be the minimal stripwise subconvex arc joining $x$ and $y$ in $D$. Since $C(x, y) \subset D$ and $D$ is contained in a two-dimensional flat $F$ parallel to $R$, we may apply the same arguments of the proof of Theorem 2 to conclude that $C(x, y)$ is a monotone arc without a horizontal line segment if $x$ and $y$ are not on a horizontal line in $F$. If $x$ and $y$ are on a horizontal line in $F \cap S$, then the stripwise subconvex $C(x, y)$ is equal to $x y$. Hence $S$ is strictly monotone.

\section{REFERENCES}

1. V. L. Klee, Convex sets in linear spaces, Duke Math. J. 18 (1951), 443-466.

2. J. Slaninger, Subconvex sets, Dissertation, Univ. of California, Los Angeles, 1970.

3. J. Chan Stanek, Tietze-type theorems on monotone increasing sets, Proc. Amer. Math. Soc. 54 (1976), 286-290.

4. H. Tietze, Über Konvexheit im kleinen und im grossen und über gewisse den Punkten einer Menge zugeordnete Dimensionzahlen, Math. Z. 28 (1928), 697-707.

5. F. A. Valentine, Convex sets, McGraw-Hill, New York, 1964.

Department of Mathematics, Sonoma State College, Rohnert Park, California 94928 